\title{
LITTLE BABIES WITH LITTLE NUMBERS: THE SIGNIFICANCE OF THROMBOCYTOPENIA IN NEONATAL INTENSIVE CARE
}

\section{A. Curley}

Cambridge University Hospitals NHS Foundation Trust, Cambridge, UK

Neonatal thrombocytopenia and bleeding are common and important clinical problems in neonatology affecting $25 \%$ of all neonatal unit admissions. Platelet transfusions are frequently used in modern neonatal clinical practice as prophylaxis in thrombocytopenic neonates. Policies and protocols for neonatal platelet transfusion therapy vary widely between clinicians and institutions, reflecting the generally broad nature of recommendations in national guidelines, which themselves are based largely on consensus rather than evidence and often extrapolated from adult data. Recent randomised trials addressing red cell transfusion triggers for anaemic neonates have contributed to the debate on appropriate neonatal red cell transfusions. However, a comparable platelet trigger trial, assessing clinically relevant outcomes, has never been undertaken in preterm neonates with severe thrombocytopenia (platelet counts $<50 \times 10^{9} / \mathrm{L}$ ). There have been concerns about adverse reactions to platelet transfusions in neonates with reports suggesting an association between repeated platelet transfusions and adverse events such as necrotising enterocolitis and sepsis. The risk of bacterial contamination is estimated at 15 per million donations but platelets are the blood component most likely to be contaminated by bacteria as they are stored at room temperature. This lecture will cover the existing evidence governing platelet transfusion practice in neonatology and outline proposed research in the area. The development of a dedicated neonatal bleeding assessment tools for use in neonatology in conjunction with platelet threshold trials will be discussed. 